\title{
Pore-scale modeling of vapor transport in partially saturated capillary tube with variable area using chemical potential
}

\author{
Addassi, Mouadh; Schreyer, Lynn; Johannesson, Björn ; Lin, Hai
}

Published in:

Water Resources Research

Link to article, DOI:

10.1002/2016WR019165

Publication date:

2016

Document Version

Publisher's PDF, also known as Version of record

Link back to DTU Orbit

Citation (APA):

Addassi, M., Schreyer, L., Johannesson, B., \& Lin, H. (2016). Pore-scale modeling of vapor transport in partially saturated capillary tube with variable area using chemical potential. Water Resources Research, 52.

https://doi.org/10.1002/2016WR019165

\section{General rights}

Copyright and moral rights for the publications made accessible in the public portal are retained by the authors and/or other copyright owners and it is a condition of accessing publications that users recognise and abide by the legal requirements associated with these rights.

- Users may download and print one copy of any publication from the public portal for the purpose of private study or research.

- You may not further distribute the material or use it for any profit-making activity or commercial gain

- You may freely distribute the URL identifying the publication in the public portal 


\section{Water Resources Research}

\section{RESEARCH ARTICLE \\ 10.1002/2016WR019165 \\ Pore-scale modeling of vapor transport in partially saturated capillary tube with variable area using chemical potential}

Key Points:

- Vapor transport through a capillary tube of variable area with liquid bridges are modeled with one equation and no fitting parameter - Relative humidity as the dependent variable does not require modeling small temperature fluctuations, surface tension, or contact angles - Enhancement factor depends on relative humidity boundary conditions, distance between liquid bridges, and bridge lengths

Supporting Information: - Supporting Information S1

Correspondence to:

L. Schreyer,

Lynn.Schreyer@wsu.edu

\section{Citation:}

Addassi, M., L. Schreyer, B. Johannesson, and H. Lin (2016), Pore-scale modeling of vapor transport in partially saturated capillary tube with variable area using chemical potential, Water Resour. Res., 52, doi:10.1002/2016WR019165.

Received 5 MAY 2016 Accepted 23 AUG 2016 Accepted article online 29 AUG 2016

(c) 2016. American Geophysical Union. All Rights Reserved.

\author{
Mouadh Addassi ${ }^{1}$ Lynn Schreyer², Björn Johannesson³, and Hai Lin 4 \\ ${ }^{1}$ Technical University of Denmark, Lyngby, Denmark, ${ }^{2}$ Washington State University, Pullman, Washington, USA, ${ }^{3}$ Linnaeus \\ University, Växjö, Sweden, ${ }^{4}$ University of Colorado Denver, Denver, Colorado, USA
}

\begin{abstract}
Here we illustrate the usefulness of using the chemical potential as the primary unknown by modeling isothermal vapor transport through a partially saturated cylindrically symmetric capillary tube of variable cross-sectional area using a single equation. There are no fitting parameters and the numerical solutions to the equation are compared with experimental results with excellent agreement. We demonstrate that isothermal vapor transport can be accurately modeled without modeling the details of the contact angle, microscale temperature fluctuations, or pressure fluctuations using a modification of the Fick-Jacobs equation. We thus conclude that for a single, axisymmetric pore, the enhancement factor depends upon relative humidity boundary conditions at the liquid bridge interfaces, distance between liquid bridges, and bridge lengths.
\end{abstract}

\section{Introduction}

Vapor transport in variably saturated soils is traditionally modeled using a system of equations including the equation representing the conservation of mass, a multiphase Darcy equation, and a version of the Philip and deVries equation [Philip and de Vries, 1957] for heat transfer. Typically, the continuity equations and multiphase Darcy equation are combined to form one equation with one unknown: usually moisture content or capillary pressure (which is equivalent to liquid pressure if the gas phase is assumed to be at constant pressure) [Bear, 1972; Moradi et al., 2015]. An alternative formulation uses the matric potential-a potential that incorporates capillary potential and adsorption [Marshall et al., 2006]. The term potentials is generalized here to include quantities that are mathematically defined as primary dependent variables. Each potential has advantages and disadvantages for quantitative analysis; for example, the capillary potential and matric potential are discontinuous at the macroscale liquid front, the capillary pressure and matric potential can vary by order of magnitudes leading to numerical instabilities, and water content in the form of saturation is not appropriate for swelling soils where $100 \%$ saturation does not give full information about water content. Use of these potentials can be difficult in more complicated situations such as threephase flow, phase transfer due to the presence of, e.g., ice, or flow through expansive soils where swelling forces add to the myriad of other microscopic forces influencing water movement. Although other potentials have been considered (chemical potential, for example, has been used in the process of developing models mechanistically [Cary, 1963; Raats, 1975; Bénet et al., 2009]), a model using alternative potentials has rarely, to these authors' knowledge, been seriously considered, perhaps because experiments are traditionally designed to measure quantities such as capillary pressure and saturation.

If we look to adjacent fields, especially building materials, we find that a wider variety of potentials are being considered for use [Janssen, 2014; Arfvidsson and Claesson, 2000; Delgado et al., 2013]. While capillary pressure and moisture content are commonly used for moisture transport through concrete and wood [e.g., Burch and Chi, 1997], other potentials are also being explored. Examples include chemical potential [Siau, 1983], logarithm of capillary pressure [Pedersen, 1992; van Schijndel, 2011], relative humidity [Bazant and Xi, 1993; Kunzel, 1995; Grasley et al., 2006; Huang et al., 2015; Johannesson and Nyman, 2010], and even the Kirchhoff potential [Arfvidsson and Claesson, 2000]. Some of the advantages and disadvantages of these potentials are outlined by Janssen [2014].

Here we describe the use of the chemical potential, which for all practical purposes is equivalent to relative humidity under isothermal conditions, and demonstrate its utility by modeling an experiment designed to 
explain the effect of isolated liquid bridges (liquid trapped within a pore structure with gas on either side and no gas connecting path) on the enhancement factor. The enhancement factor is a macroscale parameter that takes into account microscale processes that cause "enhanced" diffusion and are used in macroscopic moisture transport models that incorporate heat transfer that are based on or generalizations of the model developed by Philip and de Vries [Philip and de Vries, 1957]. Defined as the ratio of the vapor flux in a partially saturated porous media divided by the vapor flux in "dry" (very low moisture content) porous media (based on Fick's law), it has been experimentally determined to range between 3 (at low-moisture content) and 18 (at high-moisture content) for lab and field scale experiments [Gurr et al., 1952; Taylor and Cavazza, 1954; Cary, 1966; Jackson et al., 1974; Cass et al., 1984; Assouline et al., 2013]. The incorporation of an enhancement factor is common [Wildenschild and Roberts, 2001; Saito et al., 2006; Sakai et al., 2009; Gonzalez et al., 2012], although several studies have demonstrated a lack of agreement between theory and experiments [Cahill and Parlange, 1998; Smits et al., 2011; see also Shokri et al., 2009, and references therein].

The experiment we chose to simulate is conducted at the pore scale, for which most modeling approaches involve incorporating some or all of: the pressures of the liquid and gas phase, the contact angle and/or surface tension, disjoining pressure, and temperature (incorporating the latent heat of evaporation) [see, e.g., Philip, 1964; Shahraeeni and Or, 2010, 2012; Wang and Catton, 2001; Gray and Miller, 2014]. Here we conceptually explore modeling vapor transport directly using the chemical potential (relative humidity for water) as the primary unknown.

The chemical potential is a natural dependent variable because of its properties. One definition of the chemical potential is the change in Gibbs energy required to insert a mole of a species $j$ into the system keeping temperature, number of moles of other species, and pressure fixed [Castellan, 1983]. The properties of the chemical potential include that (1) it is the potential for diffusive flow [Castellan, 1983], (2) at equilibrium the chemical potentials of one component coexisting in two phases are equal [Castellan, 1983], and (3) the rate of phase change is proportional to the difference in the chemical potentials [Bennethum et al., 1996, 2000; Bénet et al., 2009].

Here we consider vapor transport through a partially saturated cylindrically symmetric capillary tube with variable cross-sectional area at constant temperature, although the results can be generalized to nonisothermal conditions (see section 4).

In the next section we derive the governing equation, and then we compare the numerical results with experimental results under a variety of conditions. We follow this with some concluding remarks.

\section{Governing Equations}

Our goal is to derive the governing equation for mass transport of water vapor in terms of the relative humidity through a cylindrically symmetric pore that may be partially saturated and whose radius varies.

We first convert the diffusive equation, typically written using concentration as the dependent variable, to one in which the relative humidity is the dependent variable. Then, we introduce the upscaled version of this equation called the Fick-Jacobs equation that accounts for changes in capillary cross-sectional area.

For this derivation we make the following assumptions:

1. The gas phase has two components-water vapor $g_{v}$ and dry air $g_{a}$.

2. The water vapor and dry air can each be considered as an ideal gas.

3. We assume that evaporation/condensation is dominated by diffusion, i.e., that the chemical potential of the liquid and gas phases at an interface are approximately equal. Thus, evaporation or condensation occurs only as the gaseous water molecules diffuse away from the interface causing water molecules to condense or evaporate as is appropriate to keep the thermodynamic equilibrium at the interface.

4. The mass-averaged velocity of the gas phase is negligible relative to the diffusion of the water vapor with respect to the gas phase [Whitaker, 1991; Bird et al., 2007].

5. Within the isolated liquid phases the pressure and concentration variations are assumed negligible.

6. The water vapor in the gas phase is sufficiently dilute that the molar mass of the gas phase can be treated as constant.

7. The temperature $T$ and gas phase pressure $p^{g}$ variations are negligible. 
We begin with the conservation of mass (continuity equation) and a generalized Fick's law to derive the governing equation in terms of relative humidity. Notation section provides details of the governing equation derivation, numerical validation of the governing equation, and details regarding the experimental geometry.

The conservation of mass assuming no loss or gain of mass except at the boundaries of the domain in terms of the density of component $j$ of the gas phase $\rho^{g_{j}}$ is given by

$$
\frac{\partial \rho^{g_{j}}}{\partial t}=-\nabla \cdot\left(\rho^{g_{j}} \mathbf{v}^{g_{j}}\right)
$$

Adding and subtracting $\nabla \cdot\left(\rho^{g_{j}} \mathbf{v}^{g}\right)$ and using assumption (4) we obtain

$$
\frac{\partial \rho^{g_{j}}}{\partial t}=-\nabla \cdot\left(\rho^{g_{j}} \mathbf{v}^{g_{j}, g}\right)
$$

where $\mathbf{v}^{g_{j}, g}=\mathbf{v}^{g_{j}}-\mathbf{v}^{g}$ is the diffusive velocity of component $j$ in the gas phase and $\mathbf{v}^{g}$ is the bulk velocity of the gas phase (see notation section).

To close the system we use a generalized version of Fick's law to eliminate the diffusive velocity. Fick's law states that diffusion is proportional to either molar or mass fraction gradient, e.g., for mass fraction [Bird et al., 2007]

$$
\rho^{g_{j}} \mathbf{v}^{g_{j}, g}=-\rho^{g} D^{g_{j}} \nabla C^{g_{j}},
$$

where $D^{g_{j}}$ is the diffusion coefficient for Fick's law, $C^{g_{j}}$ is the mass fraction, and $\rho^{g}=\rho^{g_{v}}+\rho^{g_{a}}$. However, we will use a generalized version of Fick's law, generally reserved for diffusion of a charged species [Castellan, 1983]

$$
\rho^{g_{j}} \mathbf{v}^{g_{j}, g}=-D_{\mu}^{g_{j}}\left(\frac{\rho^{g_{j}}}{R^{g_{j} T}}\right) \nabla \mu^{g_{j}},
$$

where $\mu^{g_{j}}$ is the mass-weighted chemical potential (see notation section), $T$ is (absolute) temperature, and $R^{g_{j}}$ is the specific gas constant for component $j$. As this is currently written, the units of $D_{\mu}^{g_{j}}$ has units of length ${ }^{2}$ per time.

To determine the relationship between $D_{\mu}^{g_{j}}$ and $D^{g_{j}}$ from (2), we begin with the relationship between the chemical potential of an ideal gas in a mixture $\mu^{g_{j}}$ and its molar fraction given by the ratio of the partial pressure $p^{g_{j}}$ and total pressure of the mixture $p^{g}$ [Castellan, 1983]

$$
\mu^{g_{j}}(T, p)=\mu_{*}^{g_{j}}(T, p)+R^{g_{j}} T \ln \left(\frac{p^{g_{j}}}{p^{g}}\right),
$$

where $\mu_{*}^{g_{v}}$ is the chemical potential of pure $j$ gas at pressure $p$ and temperature $T$. Assuming the water vapor and dry air can be treated as an ideal gas (Assumption (2)), we have

$$
p^{g_{j}}=R^{g_{j}} T \rho^{g_{j}} .
$$

If the water vapor is sufficiently dilute so that the molecular mass of the gas phase $M^{g}$ can be treated as a constant (Assumption (6)) we have

$$
\nabla \mu^{g_{j}}=\frac{R^{g_{j}} T}{C^{g_{j}}} \nabla C^{g_{j}}
$$

Substituting (6) into (3) to eliminate $\nabla \mu^{g_{j}}$ and then comparing with (2) we find that

$$
D_{\mu}^{g_{j}}=D^{g_{j}} .
$$

Thus for the remainder of the paper we will not distinguish between $D_{\mu}^{g_{j}}$ and $D^{g_{j}}$, and just use $D^{g_{j}}$. The reader however should note that these two diffusion coefficients are only the same when the mixture is dilute enough that the molar mass of the gas phase can be treated as a constant.

We next rewrite the continuity equation in terms of relative humidity, $\phi$. Relative humidity is defined to be the ratio between the water vapor pressure and the water vapor pressure at saturated conditions at the same temperature 


$$
\phi=\frac{p^{g_{v}}\left(\rho^{g_{v}}, T\right)}{p_{\text {sat }}^{g_{v}}(T)}=\frac{\rho^{g_{v}}\left(p^{g_{v}}, T\right)}{\rho_{\text {sat }}^{g_{v}}(T)},
$$

where the second equality is a consequence of using the ideal gas law (5). Using (7) and (4) to determine the relationship between the chemical potential and the relative humidity yields

$$
\mu^{g_{v}}=\mu_{*}^{g_{v}}+R^{g_{j}} T \ln (\phi)+R^{g_{j}} T \ln \left(\frac{p_{\text {sat }}^{g_{v}}(T)}{p^{g}}\right) .
$$

Using (8) to eliminate the chemical potential from the generalized Fick's law (3), while assuming that variations in the gas phase pressure and temperature are negligible (so that we may assume $p^{g}, T$, and $p_{\text {sat }}^{g_{v}}$ are constants), we obtain

$$
\rho^{g_{v}} \mathbf{v}^{g_{v}, g}=-D^{g_{v}} \frac{\rho^{g_{v}}}{\phi} \nabla \phi
$$

Using the ideal gas law (5) twice, we also have

$$
\frac{\partial \rho^{g_{v}}}{\partial t}=\frac{1}{R^{g_{v} T}} \frac{\partial p^{g_{v}}}{\partial t}=p_{\text {sat }}^{g_{v}} \frac{1}{R^{g_{v}} T} \frac{\partial \phi}{\partial t}=\rho_{\text {sat }}^{g_{v}} \frac{\partial \phi}{\partial t} .
$$

Using (9) and (10) into the conservation of mass (2), we have the continuity equation in terms of the relative humidity

$$
\begin{aligned}
\rho_{\text {sat }}^{g_{v}} \frac{\partial \phi}{\partial t} & =D^{g_{v}} \nabla \cdot\left(\frac{\rho^{g_{v}}}{\phi} \nabla \phi\right), \\
\frac{\partial \phi}{\partial t} & =D^{g_{v}} \nabla \cdot(\nabla \phi),
\end{aligned}
$$

where we used (7) and we again assumed that temperature changes are negligible so that $\rho_{\text {sat }}^{g_{v}}$ can be treated as a constant. Equation (11) is our governing equation.

Assuming diffusion is through a cylindrically symmetric tube with variable cross-sectional area we can use a one-dimensional approximation to the diffusion equation (11). The derivation of this equation is provided in Supporting Information. Let $A(x)$ be the cross-sectional area which varies along the longitudinal direction $x$, and define the area-averaged relative humidity as

$$
\bar{\phi}(x, t)=\frac{1}{A(x)} \int_{A(x)} \phi(x, y, z, t) d y d z .
$$

Then the Fick-Jacobs equation [Jacobs, 1967; Patlak, 1973] can be written as

$$
\frac{\partial \bar{\phi}}{\partial t}=D^{g_{v}}\left(\frac{\partial^{2} \bar{\phi}}{\partial x^{2}}+\frac{1}{A(x)} \frac{\partial \bar{\phi}}{\partial x} \frac{d A}{d x}\right) .
$$

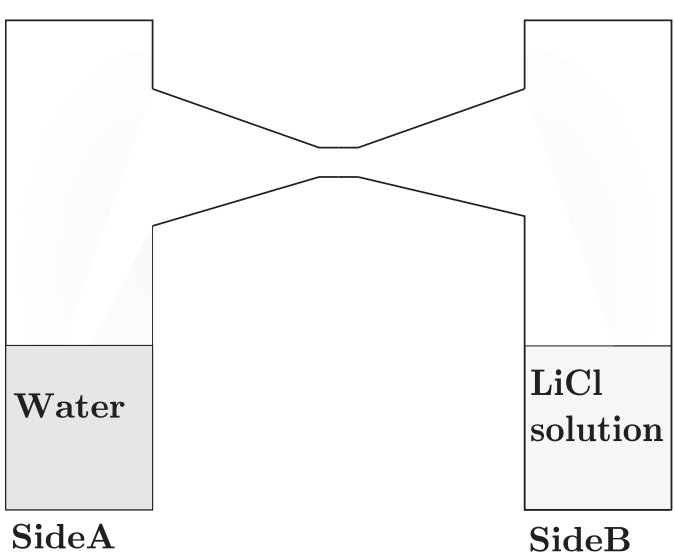

Figure 1. Experimental apparatus.
The first term on the right-hand side is the term resulting from the classical one-dimensional diffusion equation, and the second term is the modification due to changing area. This equation is the basis for further modifications, examples of which are given by Zwanzig [1992], where the diffusion coefficient $D$ is modified, and Kalinay and Percus $[2005,2006]$ where the coordinate $x$ is transformed. We numerically compared solutions of (13) with the solutions of the three-dimensional diffusion equation (11) and found the solutions to be remarkably similar (see Supporting Information).

\section{Comparison With Experiments}

In this section we use the transport equation in terms of relative humidity (equation (13)) to model 


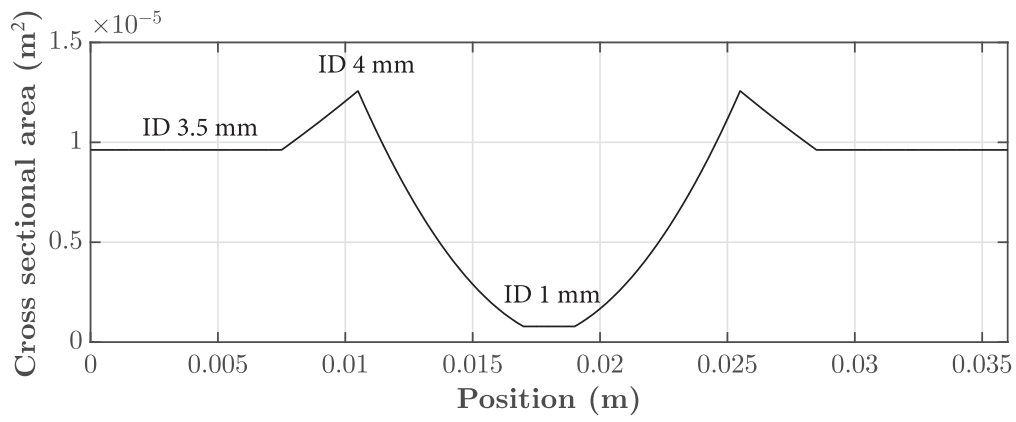

Figure 2. Cross-sectional area versus distance from base at side A.

vapor transport through a partially saturated capillary tube and compare with experimental results. Because the primary independent variable is the chemical potential (rewritten in terms of the relative humidity) which is continuous between phases at equilibrium, we do not attempt to model the air-liquid interfaces explicitly. Instead, we assume that even in the non-steady state case the vapor transport is dominated by vapor diffusion (and not evaporation or condensation) to determine how accurately we capture the physics with this simple expression. We use no fitting parameters.

The experimental data were obtained by experiments performed by T. S. Silverman for a MS thesis in 1999 [Silverman, 1999] under the supervision of John Wilson at New Mexico Institute of Mining and Technology.

\subsection{Experimental Setup}

The setup, given in Figure 1, consists of a glass apparatus where two liquid reservoirs are separated by a bridge that is either completely filled with an air-water vapor, or has a "liquid bridge." The reservoir on the left side (denoted as side A) is pure water and thus the boundary condition on the air-side of the liquid-air interface is $100 \%$ relative humidity. The reservoir on the right side is a saturated solution and thus the boundary condition on the right side (denoted as side B) is fixed at less than $100 \%$, causing water to evaporate from the left side and to condense on the right side. If a liquid bridge exists, then the water transport is affected by the length of the bridge. The rate of transport is measured by taking pictures and noting the change in height of the two columns of reservoir fluids. The goal is to determine how the length of the bridge affects the movement of water vapor and to predict the vapor transport using equation (11).

The details of the geometry are given in the Supporting Information; however, here we mention that the horizontal distance between the center of the two reservoirs is $20 \mathrm{~mm}$, the inner diameter of the two columns containing the reservoirs is $3.5 \mathrm{~mm}$, and the maximum inner diameter for the bridge component is $4 \mathrm{~mm}$ which narrows to $1 \mathrm{~mm}$ at the center. In Figure 2, we see how the cross-sectional area varies with position, as measured from the base at side $A$. The cross-sectional area narrows at the center of the horizontal member, and the cross-sectional area was approximated at the $90^{\circ}$ bends as linearly increasing between the area of the vertical members to the area of the horizontal member, neglecting the excess volume at the $90^{\circ}$ bends.

All experiments used pure water (relative humidity of 100\%) in the column at side A, while the column on the right contained a saturated $\mathrm{LiCl}$ solution (relative humidity at saturation of $11 \%$ ) with solid $\mathrm{LiCl}$ crystals at the bottom to ensure brine saturation. Because the chemical potential of a component in a mixture (water with $\mathrm{LiCl}$ ) is less than the chemical potential of the pure component (pure water), the difference in chemical potentials drives water movement (in the form of water vapor) from left to right. As can be seen from equation (8) if the gas pressure and temperature are constant, a decrease in chemical potential means the relative humidity decreases-hence we would expect the relative humidity of the air above the column containing the $\mathrm{LiCl}$ solution to be less than the relative humidity of pure water, and this is indeed the case.

Table 1. Water Activity for $\mathrm{Ca}\left(\mathrm{NO}_{3}\right)_{2}$ at $29^{\circ} \mathrm{C}$

26

85
3

80

3.5
75

4

68
4.4

63 


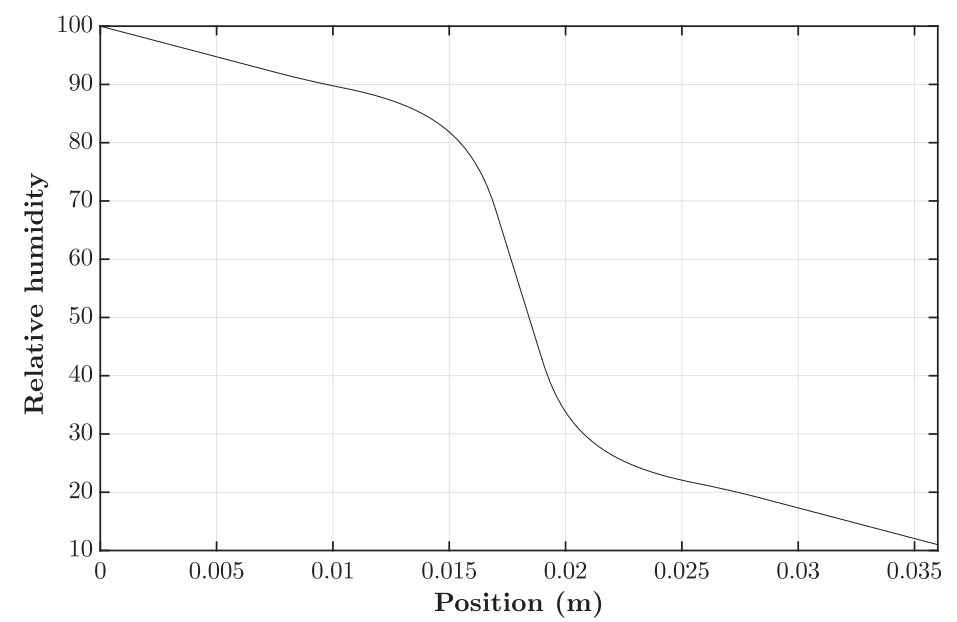

Figure 3. Numerical solution for relative humidity versus distance from base at side A with no bridge.
The relative humidity at the liquid-gas interface at side $A$ is $100 \%$, and the relative humidity at the liquid-gas interface at side $B$ is held at $11 \%$ because it is saturated. If the $\mathrm{LiCl}$ solution were to become diluted, then the chemical potential on side $B$ would increase and consequently the relative humidity boundary condition would increase with time. Likely, $\mathrm{LiCl}$ was used because of the low water chemical potential it produces when saturated and ease of access. The liquid used for the liquid bridge was a $\mathrm{Ca}\left(\mathrm{NO}_{3}\right)_{2}$ solution, and likely this solution was used because the relative humidity (or equivalently, chemical potential) at the solutiongas interface was between 11 and 100\% for a wide range of concentrations (see Table 1).

\subsection{Comparison With Data}

First consider the case of no bridge. According to equation (13), the solution at steady state would be linear if the cross-sectional area was constant, but due to the varying cross-sectional area, the solution is nonlinear.

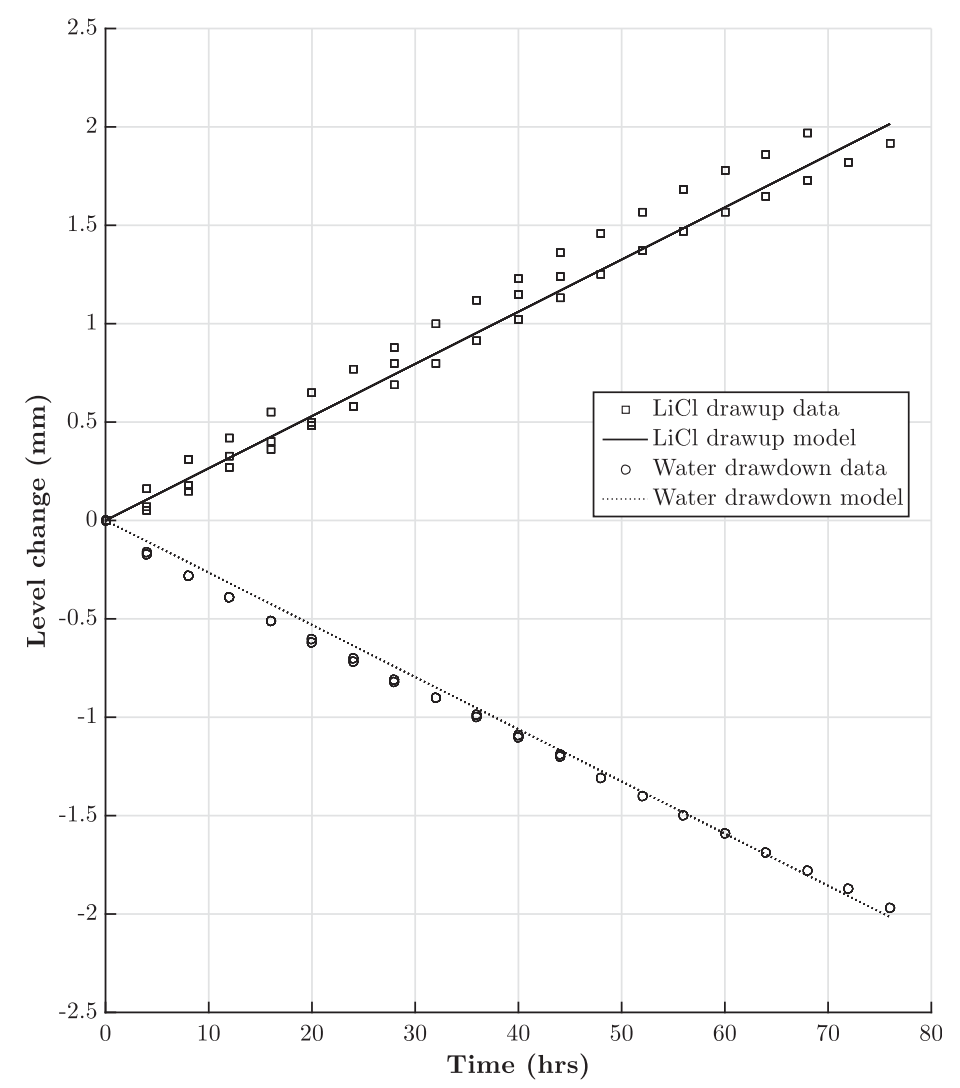

Figure 4. Silverman [1999] experimental data and numerical solution of drawdown at side $A$ and drawup at side $B$ with no bridge.
Technically, the liquid density on side $A$ is not precisely the same as the density on side $B$ due to the different concentrations. But this difference is second order and beyond the resolution of the experimental data. Here we used the steady state version of (13) with the following boundary conditions:

$$
\begin{aligned}
& \frac{d^{2} \bar{\phi}}{d x^{2}}+\frac{1}{A(x)} \frac{d \bar{\phi}}{d x} \frac{d A}{d x}=0, \\
& \bar{\phi}(0)=1, \quad \bar{\phi}(L)=0.11 .
\end{aligned}
$$

The relative humidity profile of the analytical solution is provided in Figure 3. The distance is given in meters and note that the location of the most rapid change of the relative humidity is where the crosssectional area is most narrow.

The comparison between the experimental and analytical results is provided in Figure 4 in a chamber that was roughly at a temperature of $29^{\circ} \mathrm{C}$. The line with a positive slope gives the predicted 


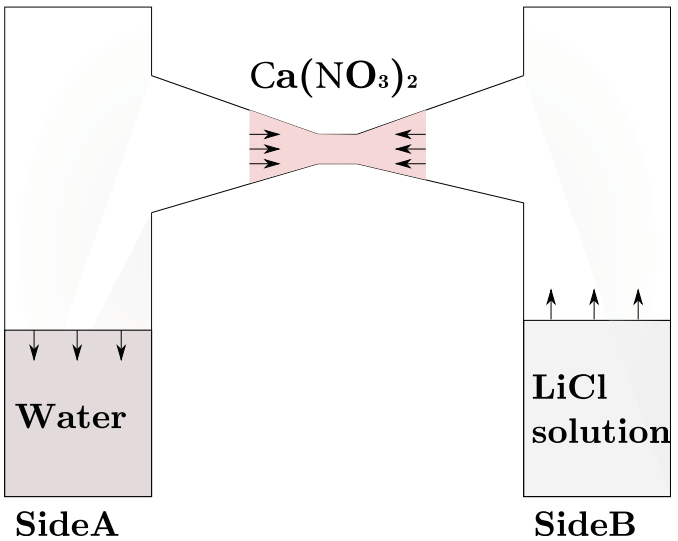

Figure 5. Experimental setup with bridge with arrows indicating direction of interfacial movement.

is inconsistency in Silverman [1999] regarding whether the initial in the text) or 2.6 molarity (as reported in the data). This affects the boundary condition on either side of the bridge with 2.6 molality resulting in a boundary condition of $71 \%$ relative humidity and $2.6 \mathrm{M}$ resulting in a boundary condition of $85 \%$ relative humidity. By studying the data in Silverman [1999, Appendix E] (using the provided initial mass of the bridge and the provided density), and that it is stated in the thesis that "the vapor pressure of a saturated calcium nitrate solution (approximately 50\%) was nearly midway between those of the two end-member" [Silverman, 1999] we concluded that the units must be the molality. This was further confirmed by the model. We used molality for all numerical experiments presented here.

The liquid pressure and concentration variation within the bridge is assumed to be negligible so that the relative humidity on either side of the bridge is assumed to be the same. However, as the experiment progresses in time there is a net change of liquid water in the bridge changing the concentration (and hence the relative humidity on either side of the bridge). The relative humidity (or activity) in equilibrium with a $\mathrm{Ca}\left(\mathrm{NO}_{3}\right)_{2}$ solution was determined using the data provided in Table 1, where the water activity for $\mathrm{Ca}\left(\mathrm{NO}_{3}\right)_{2}$ solutions at a variety of concentrations were determined by experiments conducted at the University of Colorado Denver Chemistry Department using a Water Activity Meter (using series 3TE AquaLab instrument). The concentration was measured in units of molarity (moles of $\mathrm{Ca}\left(\mathrm{NO}_{3}\right)_{2}$ per liter of water), and then converted to units of molality for use in our model.

Comparing the relative humidity gradients at the experimental conditions, we found that initially the vapor transport on the right side of the bridge (side B) is considerably higher than that of the left side of the bridge

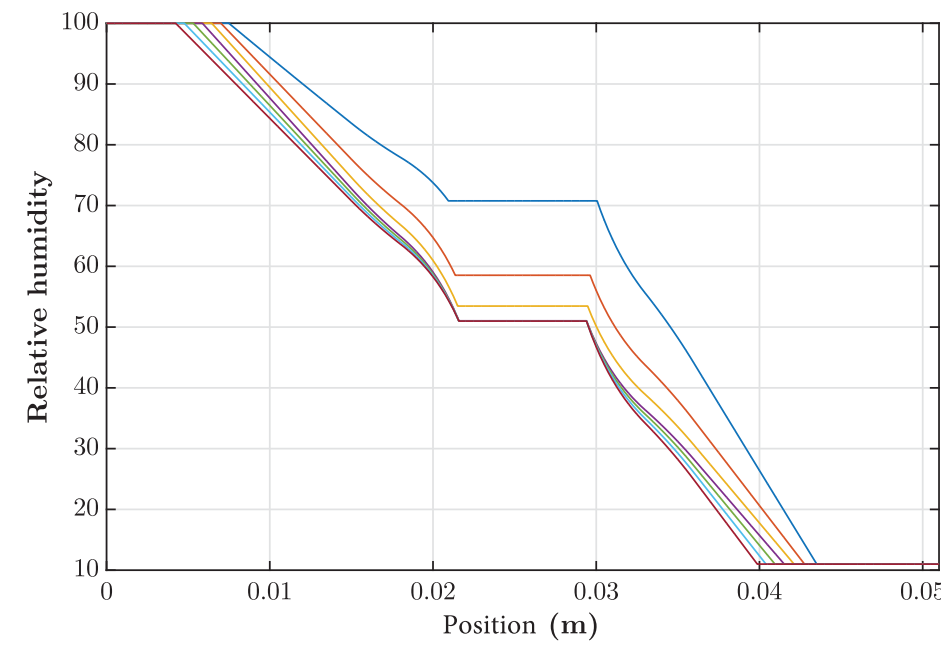

Figure 6. Numerical solution of (15)-(17) for $9.1 \mathrm{~mm}$ initial bridge length. The initial condition is in blue. (side A). Thus, we expect that the rate of condensation on the left side of the bridge is much less than the rate of evaporation on the right side of the bridge, resulting in a reduction in the length of the bridge (as noted in the experiment), a change of the bridge concentration, and a change in the relative humidity boundary conditions on either side of the bridge.

The numerical solution to this problem was obtained by solving the Fick-Jacobs equation (13), with an initial concentration of calcium nitrate in the bridge providing the initial relative humidity $\bar{\phi}_{0}$ at either side of the bridge 

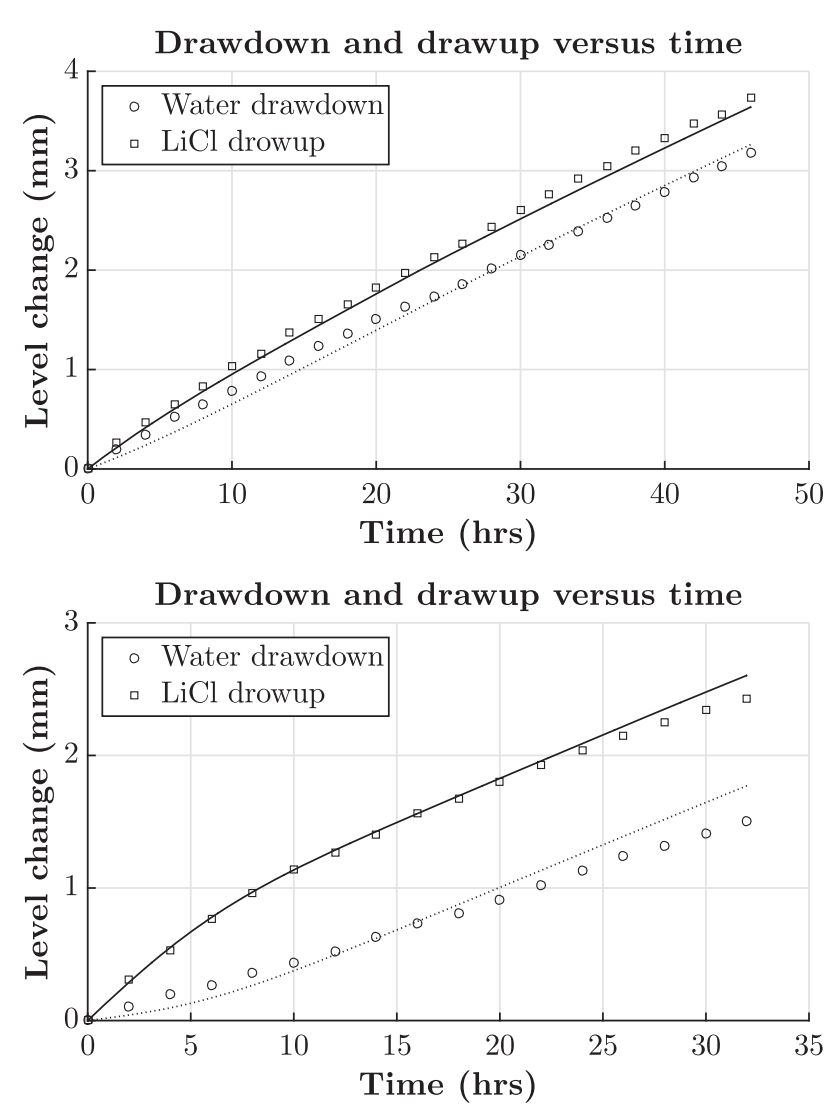
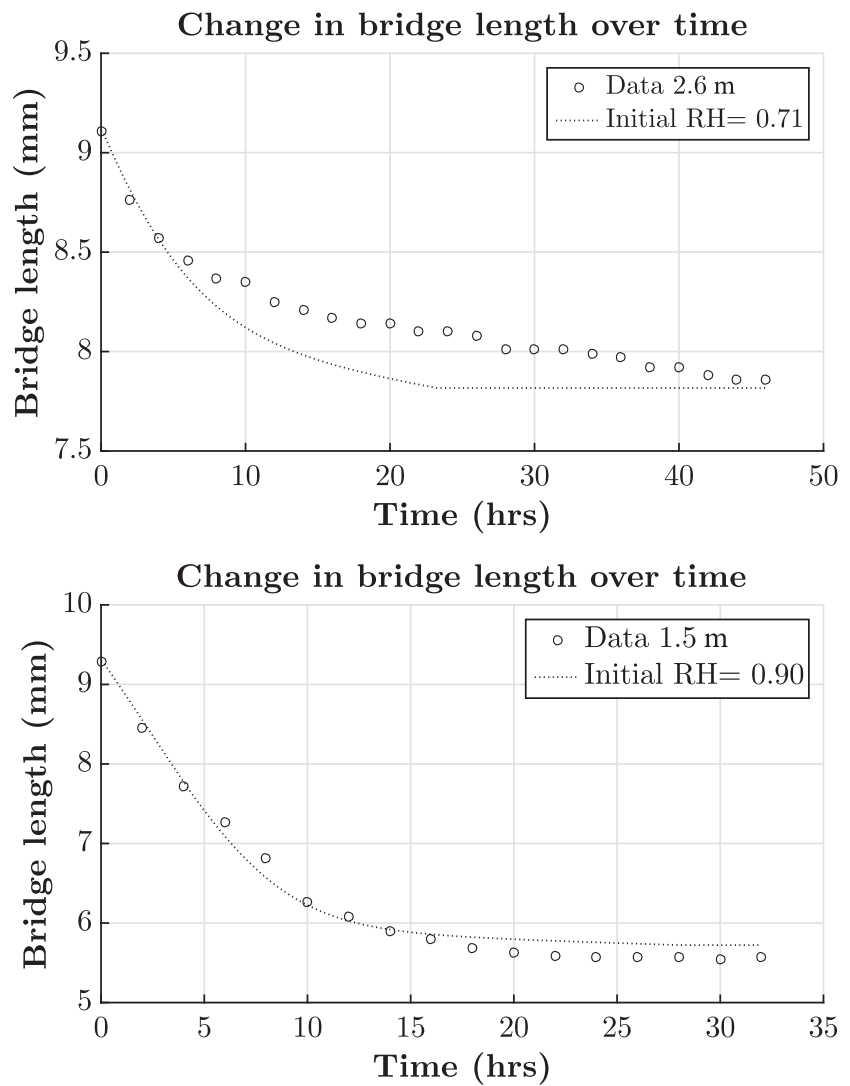

Figure 7. Numerical and Silverman [1999] data for drawdown, drawup, and change in 9.1 mm bridge length for two different bridge initial concentrations: 2.6 molality (top) and 1.5 molality (bottom).

$$
\begin{gathered}
\frac{\partial \bar{\phi}}{\partial t}=D^{g_{v}}\left(\frac{\partial^{2} \bar{\phi}}{\partial x^{2}}+\frac{1}{A(x)} \frac{\partial \bar{\phi}}{\partial x} \frac{d A}{d x}\right), \\
\bar{\phi}(x=0, t)=1, \quad \bar{\phi}(x=L, t)=.11 \quad t \geq 0, \\
\bar{\phi}(x, t=0)=\left\{\begin{array}{ll}
1 & x=x_{A}(\text { at reservoir interface Side A }) \\
\text { linear } & x \in \text { Side A } \\
0.71 & x \text { at bridge interface Side A } \\
0.71 & x \text { at bridge interface Side B } \\
\text { linear } & x \in \text { Side B }) \\
0.11 & \left.x=x_{B} \text { (at reservoir interface Side B }\right)
\end{array},\right.
\end{gathered}
$$

where "linear" refers to a linear interpolation in the region between the two interfaces.

The problem was solved as follows:

1. The change of the bridge volume is calculated using the difference in mass fluxes between side $A$ and side $B$ from the previous time step.

2. The new bridge concentration and resulting relative humidity of the bridge is calculated using the updated bridge volume.

3. The position of the liquid-gas interfaces are updated based on the mass flux of side $A$ and side $B$ from the previous time step.

The numerical result for the solution to (15)-(17) are presented in Figure 6 with $\phi_{0}=71 \%$. Note that as the solution evaporates from the left the boundary at side A moves down (or left), and conversely on the right. Over time the bridge shortens. 


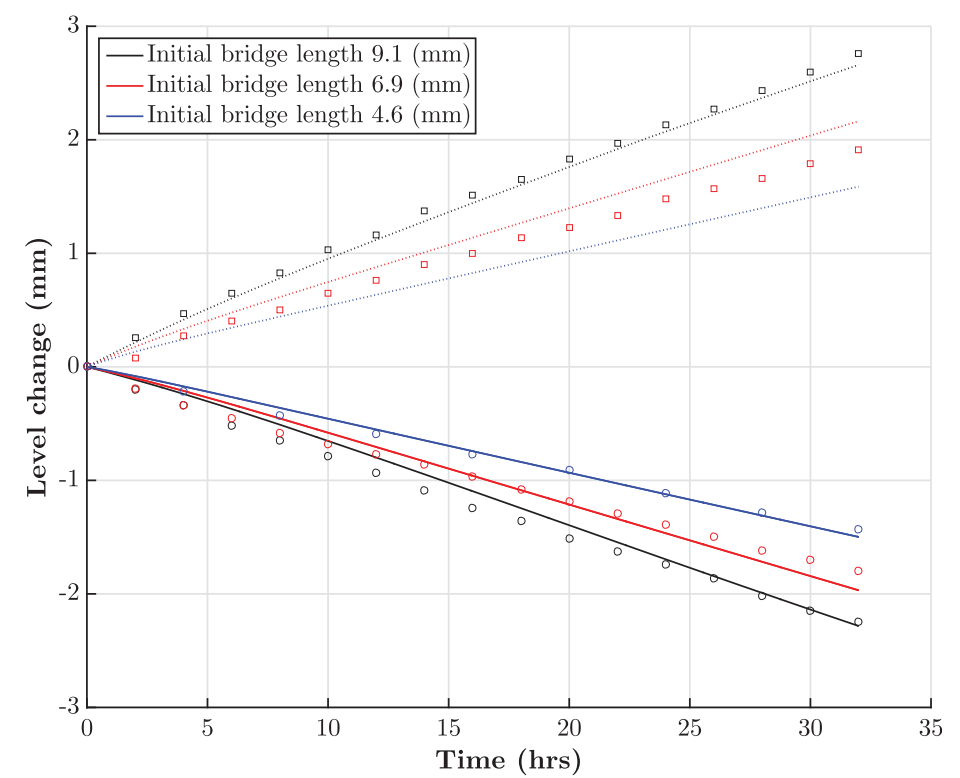

Figure 8. Numerical and Silverman [1999] experimental data for drawdown and drawup for three initial bridge lengths (no experimental data available for drawup of $4.6 \mathrm{~mm}$ length bridge).
Silverman [1999] performed the experiment with two different bridge concentrations, 2.6 molality and 1.5 molality, and the comparison with the numerical model is presented in Figure 7. Note that the lower concentration bridge (1.5 molality) which corresponds to a higher relative humidity at the bridge boundaries (90\%) results in a faster change of bridge length and a shorter steady state bridge length at steady state as can be explained by the different rates of evaporation on side $B$ due to the larger relative humidity gradient.

The comparison of experimental results with numerical results for three bridge lengths is presented in Figure 8. The initial bridge lengths were 9.1, 6.9, and $4.6 \mathrm{~mm}$.

We note that there is remarkable agreement given that there is no fitting parameter. The length measurements were made with a standard 12 inch ruler [Silverman, 1999], and as stated in Silverman [1999, p. 10] that "change in position of the meniscus in the projected image could be measured to within $0.5 \mathrm{~mm}$." So experimental and numerical differences of $0.4 \mathrm{~mm}$ would not be unexpected.

Lastly, we consider the enhancement factor. The enhancement factor was originally defined by Philip and de Vries [1957] in terms of a modified diffusion coefficient to explain the experimental speedup of diffusion in porous media where it was assumed that the enhanced diffusion was due to microscale thermal gradients and the presence of a liquid phase. It is defined as the rate of diffusion with a liquid at steady state divided by the rate of mass transfer without a liquid bridge at steady state. Silverman [1999] provides enhancement factors for varying bridge lengths and these values were used to help validate the model developed by Shahraeeni and Or [2010, 2012].

We emphasize that the enhancement factor is measured at steady state. Silverman [1999] assumed steady state was obtained when the bridge length appeared to stop changing. However we ran our numerical simulation for the case of an initial bridge length of $9.1 \mathrm{~mm}$ calculating the flux at each time step and the results are presented in Figure 9.

The top curve of Figure 9 represents the water flux on side B as measured in the rate of increase of height of side $B$ reservoir $\left(\mathrm{mm}^{3}\right.$ per hour) and the bottom dashed curve represents the flux of water evaporating from side A. At first, because of the steeper gradient on the right side, the flux on the right is much greater than the flux on the left. Somewhere around $23 \mathrm{~h}$, the bridge obtains maximum saturation (minimum humidity of about $51 \%$ at the boundaries) and the flux is limited by the flux on side Bthe water able to evaporate from the right side of the bridge cannot cause the bridge concentration to drop below saturated conditions, so the amount evaporating can only be equal to the amount condensing on the left side of the bridge. Thus, once the bridge reaches saturated conditions, the bridge length can no longer change, but the rate of evaporation from side A has not reached steady state because as the water evaporates, the length between the interface at side $A$ and the bridge increases, which ultimately decreases the flux rate. This would continue until all of the water in side A reservoir evaporates. Thus, we do not believe that a steady state was ever obtained in these experiments, making the reported enhancement factors unreliable.

However for comparison, we calculated the analytical enhancement factor under the following assumptions: (1) the height of the reservoir columns on sides A and B are both fixed at $7.5 \mathrm{~mm}$, so that the distance 


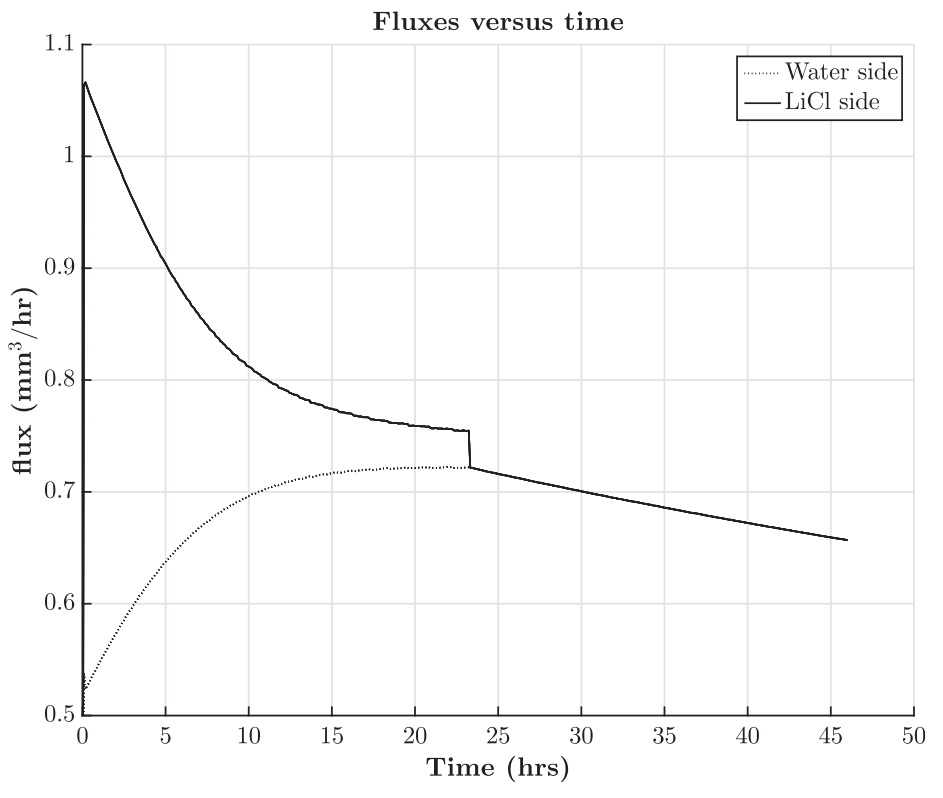

Figure 9. Numerical drawdown and drawup results for $9.1 \mathrm{~mm}$ bridge. The bridge reaches maximum concentration at approximates $23 \mathrm{~h}$ and after that the bridge length does not change. between the reservoir and gas interfaces is $36 \mathrm{~mm}$. (2) The bridge is assumed to have a $\mathrm{Ca}\left(\mathrm{NO}_{3}\right)_{2}$ concentration so that the boundary condition on either side of the bridge is a relative humidity of $55 \%$, which gives equal flux on either side. These two assumptions provide a steady state within this system and these results are compared with those reported in Silverman [1999] as a function of bridge length. Note that the model gives a higher enhancement rate because the distance between the bridge and the water-gas interface at side $A$ is shorter than that in the actual experiment, providing a larger flux of water vapor. This does give an idea of how the enhancement factor can

change depending upon the location of the bridges (how far apart liquid water sources are from each other) and the length of bridges.

\section{Conclusion and Discussion}

In this paper we took a first foray into using chemical potential to model vapor flow through partially saturated soils by demonstrating the conceptual advantage of using the chemical potential in modeling vapor transport at the pore scale. The governing equation, the Fick-Jacobs equation (13), is a one-dimensional equation for the area-averaged concentration (or relative humidity), assuming cylindrically symmetric geometry. This model is conceptually simple, requiring only the geometry and the relative humidity (or more generally the chemical potential) at the gas-liquid interfaces. The other known model used to capture this experiment was developed by Shahraeeni and $\operatorname{Or}[2010,2012]$. It incorporates temperature fluctuations

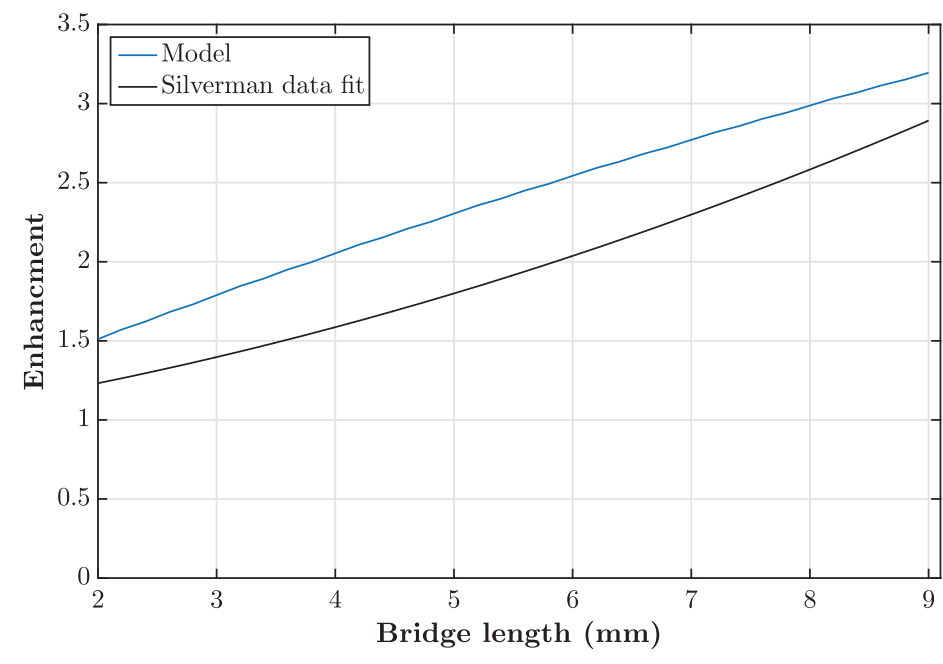

Figure 10. Enhancement calculated by Silverman [1999] using a data fit, and from numerical results for different length bridges. The difference is most likely due to the fact that the experimental system does not reach a steady state. via the heat equation, interfacial surface tension, contact angle, and rate of condensation and evaporation equations. Although much more complex, it does not require assuming constant temperature and diffusion-limited (not limited by phase-change) conditions for vapor transport.

We emphasize that there are no fitting parameters used to compare the analytical and experimental results. The most sensitive parameter in this model is the initial relative humidity at the gas-liquid interface-a quantity directly measuring chemical potential under 
isothermal conditions, which is the energy required to remove a unit amount of water from the liquid phase. We were able to explain conceptually why the bridge lengthened and why the flux of water vapor was greater on one side than the other. We then discussed the enhancement factor explaining that the data given by Silverman [1999] were probably not at steady state conditions. We argued that a constant enhancement factor for this particular experimental setup does not exist because of the constantly changing distance between the bridge interfaces and the reservoir interfaces. However, we were able to numerically calculate an enhancement factor as a function of bridge length under the condition that the reservoirgas interfaces did not move. These results are presented in Figure 10. In summary, we can state that the enhancement factor is a function of (at minimum) distance between liquid bridges, relative humidity boundary conditions at the bridge interfaces, and bridge lengths.

Because chemical potential is the most general potential for diffusive flow and phase transformation [Castellan, 1983; Bennethum et al., 1996, 2000], it provides a framework for generalizing to more complex vapor flow problems. Let us first consider the case where the surface is highly hydrophilic, creating a larger surface tension, higher radius of curvature, and a lower relative humidity at the interface. Although we might not know directly how the chemical potential changes as a function of the relative affinity of the liquid to the solid over gas, the relative humidity is directly measurable and gives an indication of its effect. Using the same geometry (e.g., capillary tube with fixed radius), and different materials making up the capillary tube, one could determine how the chemical potential changes by measuring the relative humidity at the liquidgas interface. In fact, for the case of a capillary tube, the relationship between surface tension, $\gamma$, and relative humidity, $\phi$, is already known by the Kelvin equation [Haynes, 1973; Lu and Likos, 2001]

$$
\ln \phi=\frac{2 \gamma V_{m}}{r R T}
$$

where $V_{m}$ is the molar volume of the liquid, $r$ is the radius of curvature of the interface from within the water phase (defined to be infinity if flat), and $R$ is the universal gas constant. The Kelvin equation is the basis for determining pore size distribution using adsorption porosimetry [Haynes, 1973; Wu et al., 2014]. In the rare case that the radius of curvature is greater than zero (created by a nonwetting surface) then the relative humidity at the interface is greater than 1 and the gas phase is supersaturated with water vapor (although this condition would likely not last if the pore is connected to gas at atmospheric conditions or there were other places where the water vapor could condense at a lower relative humidity). When $\phi=1$ the gas phase is saturated and the radius of curvature is infinity, and as the radius of curvature increases in the negative direction (created by a hydrophilic surface), the relative humidity at the liquid-gas interface decreases, meaning that condensation occurs at low relative humidities (the water prefers the liquid phase over the gas phase more so than when the interface is flat). We note that an order-of-magnitude analysis indicates that a relative humidity of .999 at an interface with pure liquid water at equilibrium occurs at approximately $r=1 \mathrm{~mm}$. So neglecting curvature for capillary tubes of the size in the experiment modeled here in determining the relative humidity boundary conditions is justified.

Thermal gradients are of great importance in some applications such as soil borehole thermal energy storage (SBTES) systems [Bear et al., 1991; Moradi et al., 2015]. So consider a pore-scale experiment where we no longer have isothermal conditions. Using the principle that flow is determined by the gradient of chemical potential [Castellan, 1983], we would derive the governing equation beginning with equation (3). We can still use equation (9) for chemical potential, but in calculating the gradient of the chemical potential needed for (3) we would invoke the chain and product rules so that we have a sum of a term involving the gradient of relative humidity and a gradient of temperature.

And if rate of evaporation/condensation is not dominated by diffusion [e.g., Bénet et al., 2009], we would not be able to assume that the chemical potential of water at an interface is equal in the gas and liquid phases. However, fundamental physics dictates that the rate of evaporation is proportional to the difference of the chemical potentials in the two phases [Bennethum et al., 1996, 2000; Bénet et al., 2009; Alexandersson et al., 2016]. This is in fact a generalization of what is sometimes used: the rate of phase change is based on the difference between the gas vapor pressure and the equilibrium pressure at the water-gas interface [Zhang and Datta, 2004; Smits et al., 2011; Moradi et al., 2015]. Thus, using the chemical potential as a primary unknown could directly determine the rate of phase change [see, e.g., Bénet et al., 2009]. 
Whether chemical potential can be useful as a primary unknown for hydrological modeling at the macroscale is still unknown, but its potential benefits are clear, as indicated here and as suggested by its use in other fields. Further, we believe that understanding of the chemical potential could explain more clearly transport processes. Even under fully saturated conditions, flow is determined by the chemical potential (for a single component fluid, the chemical potential is the Gibbs potential, with pressure and temperature as the primary independent variables, leading to a generalized Darcy's law [Bennethum and Cushman, 2002; Schreyer-Bennethum, 2012].

\section{Notation}

\section{Superscripts, Subscripts, and Others}

$-\quad$ area-averaged (see, e.g., equation (12)).

.9 gas phase.

.$g_{j} \quad$ component $j$ of the gas phase, $j=v$ for water vapor, and $j=a$ for dry air.

.$_{*} \quad$ pure component $j$ in gas phase.

$g_{v}$
sat

\section{Latin Symbols}

$A(x) \quad$ cross-sectional area as a function of position $x$ [length $\left.{ }^{2}\right]$.

$C^{g_{j}} \quad$ mass fraction of component $j$ in gas phase, $\rho^{g_{j}} / \rho^{g}$.

$D \quad$ diffusion coefficient for Fick's law (see equation (2)) [length ${ }^{2} /$ time] .

$D_{\mu}^{g_{j}} \quad$ diffusion coefficient for generalized Fick's law (see equation (3)) [length ${ }^{2} /$ time]. $^{2}$

$M^{g_{j}} \quad$ molecular mass of component $j$ in the gas phase [mass/mole].

$p^{g}, p_{j}^{g} \quad$ pressure of gas phase mixture, partial pressure of component $j$ in gas phase [force/area].

$R \quad$ universal gas constant [energy/(mole-temperature)].

$R^{g_{j}} \quad$ specific gas constant for component $j, R^{g_{j}}=R / M^{g_{j}}$ [energy/(mass-temp)].

$T \quad$ absolute temperature [temperature].

$\mathbf{v}^{g}, \mathbf{v}^{g_{j}} \quad$ velocity of the gas phase or component $j$ of the gas phase, $\rho^{g} \mathbf{v}^{g}=\rho^{g_{j}} \mathbf{v}^{g_{j}}+\rho^{g_{a}} \mathbf{v}^{g_{a}}$ [length/time].

$\mathbf{v}^{g_{j}, g} \quad$ diffusive velocity, $\mathbf{v}^{g_{j}}-\mathbf{v}^{g}$.

\section{Greek Symbols}

$\mu^{g_{j}} \quad$ mass-weighted chemical potential of component $j$ in gas phase [energy/mass].

$\rho^{g}, \rho^{g_{j}} \quad$ mass density of gas phase [mass of $g$ per volume of $g$ ] mass density of component $j$ in the gas phase [mass of $g_{j}$ per volume of $g$ ].

$\phi \quad$ relative humidity (see equation (7)).

Acknowledgments

Thanks to INGENIOR CAND POLYT ERIK HEGENHOFTS LEGAT for supporting Addassi's visit to the Department of Mathematical and Statistical Sciences at the University of Colorado Denver, without which this research would not have been possible. Experimental data obtained for this article are published in Table 1. Code developed to solve numerically the governing equations presented in this manuscript can be obtained by e-mailing Mouadh Addassi at addassimouadh@gmail.com.

\section{References}

Alexandersson, M., H. Askfelt, and M. Ristinmaa (2016), Triphasic model of heat and moisture transport with internal mass exchange in paperboard, Transp. Porous Media, 112(2), 381-408, doi:10.1007/s11242-016-0651-9.

Arfvidsson, J., and J. Claesson (2000), Isothermal moisture flow in building materials: Modelling, measurements and calculations based on Kirchhoff's potential, Building Environ., 35, 519-536.

Assouline, S., S. W. Tyler, J. S. Selker, I. Lunati, C. W. Higgins, and M. B. Parlange (2013), Evaporation from a shallow water table: Diurnal dynamics of water and heat at the surface of drying sand, Water Resour. Res., 49, 4022-4034, doi:10.1002/wrcr.20293.

Bazant, Z. P., and Y. Xi (1993), Stochastic drying and creep effects in concrete structures, J. Struct. Eng. ASCE, 119(1), $301-322$.

Bear, J. (1972), Dynamics of Fluids in Porous Media, Dover, N. Y.

Bear, J., J. Bensabat, and A. Nir (1991), Heat and mass transfer in unsaturated porous media at a hot boundary: I. One-dimensional analytic model, Transp. Porous Media, 6(3), 281-298.

Bénet, J.-C., A.-L. Lozano, F. Cherblanc, and B. Cousin (2009), Phase change of water in a hygroscopic porous medium. Phenomenological relation and experimental analysis for water in soil, J. Non-Equilibrium Thermodyn., 34(2), 133-153.

Bennethum, L. S., and J. H. Cushman (2002), Multicomponent, multiphase thermodynamics of swelling porous media with electroquasistatics: Il. Constitutive theory, Transp. Porous Media, 47(3), 337-362.

Bennethum, L. S., M. A. Murad, and J. H. Cushman (1996), Clarifying hybrid mixture theory and the macroscale chemical potential for porous media, Int. J. Eng. Sci., 34(2), 125-145.

Bennethum, L. S., M. A. Murad, and J. H. Cushman (2000), Macroscale thermodynamics and the chemical potential for swelling porous media, Transp. Porous Media, 39(2), 187-225.

Bird, R. B., W. E. Stewart, and E. N. Lightfoot (2007), Transport Phenomena, John Wiley, N. Y.

Burch, D. M., and J. Chi (1997), MOIST: A PC program for predicting heat and moisture transfer in building envelopes: Release 3.0, Tech. Rep. Spec. Publ. 917, Dep. of Commer., Natl. Inst. of Stand. and Technol., Gaithersburg, Md.

Cahill, A. T., and M. B. Parlange (1998), On water vapor transport in field soils, Water Resour. Res., 34(4), 731-739.

Cary, J. W. (1963), Onsager's relation and the non-isothermal diffusion of water vapor, J. Phys. Chem., 67, 126-129. 
Cary, J. W. (1966), Soil moisture transport due to thermal gradients: Practical aspects, Soil Sci. Soc. Am. Proc., 30(4), 428-433.

Cass, A., G. Campbell, and T. Jones (1984), Enhancement of thermal water vapor diffusion in soils, Soil Sci. Soc. Am. J., 48(1), 25-32, doi: 10.2136/sssaj1984.03615995001800040001x.

Castellan, G. W. (1983), Physical Chemistry, The Benjamin/Cummings Publ. Co, Menlo Park, Calif.

Delgado, J. M. P. Q., E. Barreira, N. M. M. Ramos, and V. P. de Freitas (2013), Hydrothermal Numerical Simulation Tools Applied to Building Physics, Springer, N. Y.

Gonzalez, R. G., A. Verhoef, P. L. Vidale, and I. Braud (2012), Incorporation of water vapor transfer in the JULES land surface model: Implications for key soil variables and land surface fluxes, Water Resour. Res., 48, W05538, doi:10.1029/2011WR011811.

Grasley, Z., D. Lange, and M. D'Ambrosia (2006), Internal relative humidity and drying stress gradients in concrete, Mater. Struct., 39, 901-909.

Gray, W. G., and C. Miller (2014), Introduction to the Thermodynamically Constrained Averaging Theory for Porous Medium Systems, Springer, Berlin.

Gurr, C., T. Marshall, and J. Hutton (1952), Movement of water in soil due to a temperature gradient, Soil Sci., 74(5), 335-346.

Haynes, J. (1973), Pore size analysis according to the Kelvin equation, Mater. Construct., 6(3), 209-213.

Huang, Q., Z. Jiang, X. Gu, W. Zhang, and B. Guo (2015), Numerical simulation of moisture transport in concrete based on a pore size distribution model, Cement Concrete Res., 67, 31-43.

Jackson, R. D., R. J. Reginato, B. A. Kimball, and F. S. Nakayama (1974), Diurnal soil-water evaporation: Comparison of measured and calculated soil-water fluxes, Soils Sci. Soc. Am. Proc., 38(6), 861-866.

Jacobs, M. H. (1967), Diffusion Processes, Springer, N. Y.

Janssen, H. (2014), Simulation efficiency and accuracy of different moisture transfer potentials, J. Building Performance Simul., 7(5), 379-389.

Johannesson, B., and U. Nyman (2010), A numerical approach for non-linear moisture flow in porous materials with account to sorption hysteresis, Transp. Porous Media, 84(3), 735-754.

Kalinay, P., and J. K. Percus (2005), Extended Fick-Jacobs equation: Variational approach, Phys. Rev. E, 72, 061203, doi:10.1103/ PhysRevE.72.061203.

Kalinay, P., and J. K. Percus (2006), Exact dimensional reduction of linear dynamics: Application to confined diffusion, J. Stat. Phys., 123(5), 1059-1069.

Kunzel, H. (1995), Simultaneous heat and moisture transport in building components: One- and two-dimensional calculations using simple parameters, PhD thesis, Fraunhofer Inst. of Building Phys., Stuttgart, Germany.

Lu, N., and W. J. Likos (2001), Unsaturated Soil Mechanics, John Wiley, N. Y.

Marshall, T., J. Holmes, and C. Rose (2006), Soil Physics, Cambridge Univ. Press, Cambridge, U. K.

Moradi, A., K. M. Smits, J. Massey, A. Cihan, and J. McCartney (2015), Impact of coupled heat transfer and water flow on soil borehole thermal energy storage (SBTES) systems: Experimental and modeling investigation, Geothermics, 57, 56-72.

Patlak, C. K. (1973), An approximation diffusion equation for a long narrow channel with varying cross-sectional area, Bull. Math. Biol., 35, 81-86.

Pedersen, C. R. (1992), Prediction of moisture transfer in building constructions, Building Environ., 27(3), 387-397.

Philip, J. R. (1964), Kinetics of capillary condensation in wedge-shaped pores, J. Chem. Phys., 41(4), 911-916.

Philip, J. R., and D. A. de Vries (1957), Moisture movement in porous materials under temperature gradients, Trans. AGU, 38(2), $222-232$.

Raats, P. (1975), Transformations of fluxes and forces describing the simultaneous transport of water and heat in unsaturated porous media, Water Resour. Res., 11(6), 938-942.

Saito, H., J. Simunek, and B. Mohanty (2006), Numerical analysis of coupled water, vapor, and heat transport in the vadose zone, Vadose Zone J., 5(2), 784-800, doi:10.2136/vzj2006.0007.

Sakai, H., N. Toride, and J. Simunek (2009), Water and vapor movement with condensation and evaporation in a sandy column, Soil Sci. Soc. Am. J., 73(3), 707-717, doi:10.2136/sssaj2008.0094.

Schreyer-Bennethum, L. S. (2012), Macroscopic flow potentials in swelling porous media, Transp. Porous Media, 94, 47-68.

Shahraeeni, E., and D. Or (2010), Pore-scale analysis of evaporation and condensation dynamics in porous media, Langmuir, 26(17), 13,924$13,936$.

Shahraeeni, E., and D. Or (2012), Pore scale mechanisms for enhanced vapor transport through partially saturated porous media, Water Resour. Res., 48, W05511, doi:10.1029/2011WR011036.

Shokri, N., P. Lehmann, and D. Or (2009), Critical evaluation of enhancement factors for vapor transport through unsaturated porous media, Water Resour. Res., 45, W10433, doi:10.1029/2009WR007769.

Siau, J. F. (1983), Chemical potential as a driving force for nonisothermal moisture movement in wood, Wood Sci. Technol., 17, 101-105.

Silverman, T. S. (1999), A pore-scale experiment to evaluate enhanced vapor diffusion in porous media, MS thesis, 126 pp., Purdue Univ., Socorro, N. M.

Smits, K., A. Cihan, T. Sakaki, and T. Illangasekare (2011), Evaporation from soils under thermal boundary conditions: Experimental and modeling investigation to compare equilibrium and nonequilibrium-based approaches, Water Resour. Res., 47, W05540, doi:10.1029/ 2010 WR009533.

Taylor, S., and L. Cavazza (1954), The movement of soil moisture in response to temperature gradients, Soil Sci. Soc. Am. J., 18(4), 351-358, doi:10.2136/sssaj1954.03615995001800040001x.

van Schijndel, A. (2011), Multiphysics modeling of building physical constructions, Building Simul., 4, 49-60.

Wang, J., and I. Catton (2001), Enhanced evaporation heat transfer in triangular grooves covered with a thin fine porous layer, Appl. Therm. Eng., 21, 1721-1737.

Whitaker, S. (1991), Role of the species momentum equation in the Stefan diffusion tube, Ind. Eng. Chem. Res., 30, 978-983.

Wildenschild, D., and J. Roberts (2001), Experimental tests of enhancement of vapor diffusion in topopah spring tuff, J. Porous Media, 4(1), 1-13, doi:10.1615/JPorMedia.v4.i1.10.

Wu, M., B. Johannesson, and M. Geiker (2014), Application of water vapor sorption measurements for porosity characterization of hardened cement pastes, Construct. Building Mater., 66, 621-633.

Zhang, J., and A. K. Datta (2004), Some considerations in modeling of moisture transport in heating of hygroscopic materials, Drying Technol., 22(8), 1983-2008.

Zwanzig, R. (1992), Diffusion past an entropy barrier, J. Phys. Chem., 96(10), 3926-3930. 